University of Northern lowa

UNI ScholarWorks

Faculty Publications

Faculty Work

1992

\title{
Real and Virtual Images Using a Classroom Hologram
}

Dale W. Olson

University of Northern lowa

Let us know how access to this document benefits you

Copyright (C) 1992 The American Institute of Physics. The copyright holder has granted permission for posting.

Follow this and additional works at: https://scholarworks.uni.edu/phy_facpub

Part of the Physics Commons

\section{Recommended Citation}

Olson, Dale W., "Real and Virtual Images Using a Classroom Hologram" (1992). Faculty Publications. 1. https://scholarworks.uni.edu/phy_facpub/1

This Article is brought to you for free and open access by the Faculty Work at UNI ScholarWorks. It has been accepted for inclusion in Faculty Publications by an authorized administrator of UNI ScholarWorks. For more information, please contact scholarworks@uni.edu. 


\title{
Real and Virtual
}

\section{Images Using a Classroom Hologram}

\author{
By Dale W. Olson
}

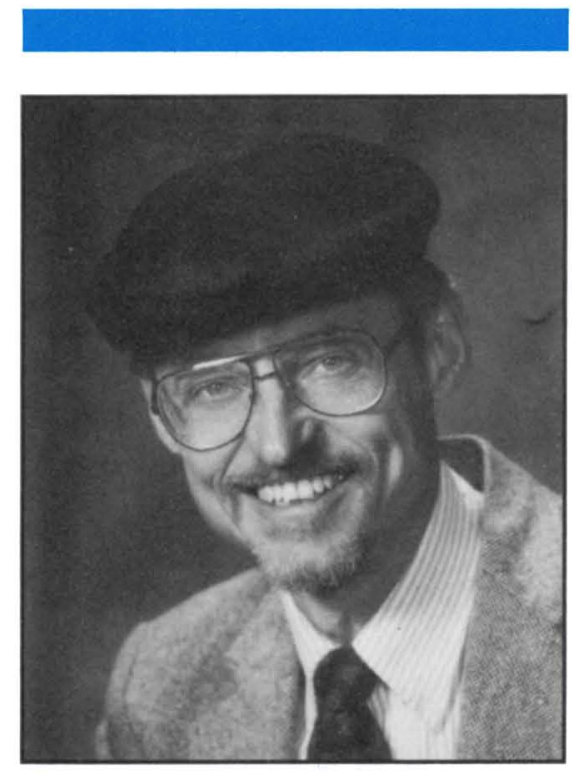

Dale Olson is associate professor of physics at the University of Northern Iowa (Cedar Falls, IA 50614-0150). He received a B.A. degree from Carleton College (Northfield, MN) in 1962 and a Ph.D. in solid state physics from the University of Rochester (Rochester, NY) in 1970. He is interested in using holography as a way of teaching principles of physics, and is also doing research on using scattered laser light and holography to measure and inspect rough surfaces.
$\mathbf{R}$ eal and virtual images appear to be frequently misunderstood by beginning physics students; a sample misunderstanding is the belief that all real images will be inverted since real images produced by lenses are always inverted. Misunderstandings related to virtual image formation also exist; an example is given later. These erroneous beliefs develop, at least in part, because students have a limited range of experience. If, as many physics education researchers believe, misunderstandings of physics concepts are best overcome by direct experience with phenomena, then exposing students to the rich and exciting imaging phenomena provided by holograms may prove valuable.

Real and virtual images are traditionally explored in introductory physics laboratories with the aid of lenses and/or mirrors; adding hologram image activities can broaden students' range of experience in a convenient, interesting, and useful way.

The activities with hologram real and virtual images described here are primarily qualitative rather than quantitative, although measurements of image distances are required. The qualitative nature of these activities contrasts sharply with traditional quantitative introductory laboratories on lens images. Such quantitative labs are important and widely used; ${ }^{1}$ they typically emphasize numerical predictions of image location by using a two-ray graphical procedure and the thin-lens equation. Very similar quantitative models also exist for thin transmission hologram images. The most elementary graphical ray tracing procedure for this type of hologram was first described by N. Abramson. ${ }^{2}$ I discussed this procedure in 1989 in the American Journal of Physics. ${ }^{3}$ In contrast, the hologram-based activities described here emphasize non-numerical activities that require no quantitative predictions of image distances from the hologram. Rather than predicting numerical values for the image distances, students draw qualitative ray diagrams; the ray diagrams link converging and diverging ray bundles to real and virtual images. Drawing qualitative ray diagrams may, on the surface, appear very elementary; in fact these drawings reveal student misconceptions about virtual images.

The hologram image activities described in this article have been tried both at high-school and introductory-college levels. I have used these procedures in two introductory college physics lab courses at the University of Northern Iowa (UNI); one lab is associated with an algebra-based introductory college physics course and the other is part of a sophomore-level course on holograms and lasers. Junior-high and senior-high teachers have tried these materials in workshops offered at UNI, including some workshops offered through the nationally based PRISMS program. Three high-school teachers have made extensive use of these materials in their physics and holography courses and report success. 


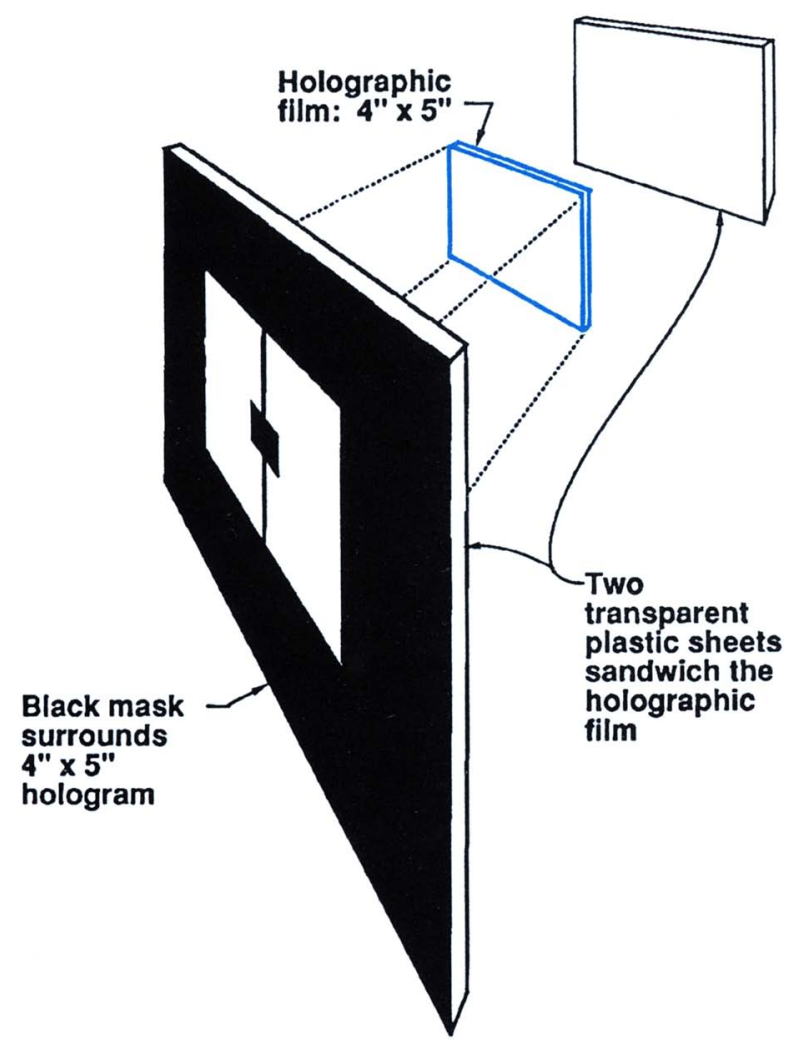

Fig. 1. Components of the classroom hologram package provided to students.

\section{Apparatus: The Classroom Hologram}

To carry out the particular activities on hologram real and virtual images described in this article, students must receive a specially fabricated classroom hologram; one such hologram is required for every three students. A suitable hologram can be made by physics teachers or as a project by a student; the required procedures are discussed later. (We are also seeking a publisher for this classroom hologram and lab activity. Todd Van Kooten, a UNI art department graduate, contributed significantly to the design and fabrication of the classroom holograms we use.)

The 4- $\times 5$-in classroom hologram is recorded on plasticbacked emulsion and sandwiched between two $8-\times 10$-in sheets of clear plastic, as shown in Fig. 1; a main function of the two plastic sheets is controlling stray laser light and preventing glare. Stray light is prevented from passing through the region immediately surrounding the hologram by a mask, as shown in Fig. 1. Stray light must also be prevented from passing through a small rectangular "window" at the center of the hologram (which exists because of the way the hologram is formed); this window is covered by a small opaque plastic rectangle also visible in Fig. 1. In addition to controlling stray light, the two plastic sheets hold and protect the film and provide ease of handling. If a disassembled hologram package is shown to the class, students are able to distinguish the holographic film from the plastic packaging.
The classroom hologram must be illuminated by a suitable point light source, which can be either a laser, as assumed in Fig. 2, or a point source of white light filtered by a narrowband interference filter. If a laser source is selected, laser safety needs to be considered in several ways. First the power of the helium-neon laser beam is limited to the range 0.5 to $3.0 \mathrm{~mW}$ of light power. Second, the laser beam is spread with a lens, with the lens preferably attached directly to the laser. Finally, the laser beam intensity can be limited by placing a light-absorbing filter in the beam. Since only $0.5 \mathrm{~mW}$ of helium-neon laser light power is required for each hologram, laser beams more powerful than $1.0 \mathrm{~mW}$ can be split and used to illuminate several classroom holograms. To guarantee that students have high-quality and safely lit images, the holograms and light sources are arranged before students arrive in the lab.

The specially fabricated classroom hologram has been designed to produce four carefully arranged image groups; these image group locations are shown in Fig. 2. Two of the image groups portray the symbols " 3 " and " $D$ " and are labeled $I_{1}$ and $I_{1}{ }^{\prime}$ in Fig. 2 ; the other two image groups portray dice and a hex-nut, and are labeled $I_{2}$ and $I_{2}{ }^{\prime}$ in Fig. 2 . Three of the four image groups are shown in perspective in Fig. 3. A fourth image group, one of the dice/hex-nut images, is not

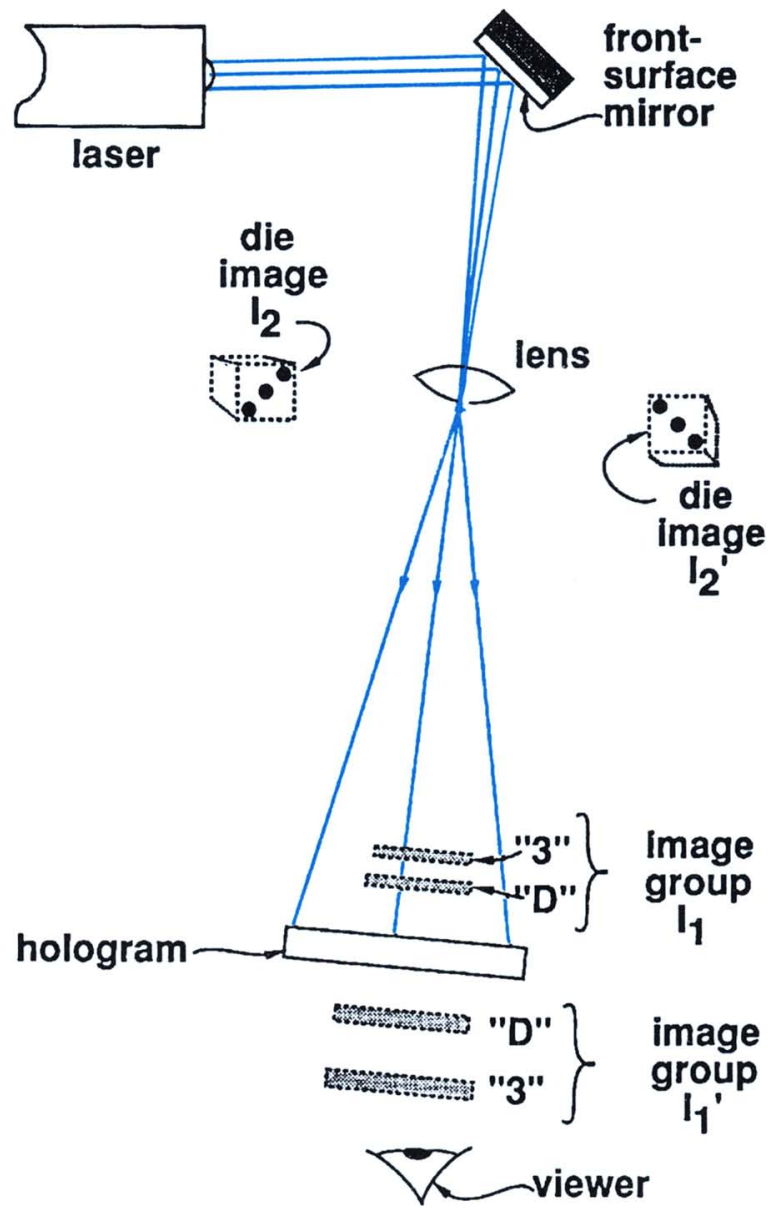

Fig. 2. Experimental arrangement used by students, showing locations of the four image groups formed by the hologram. 


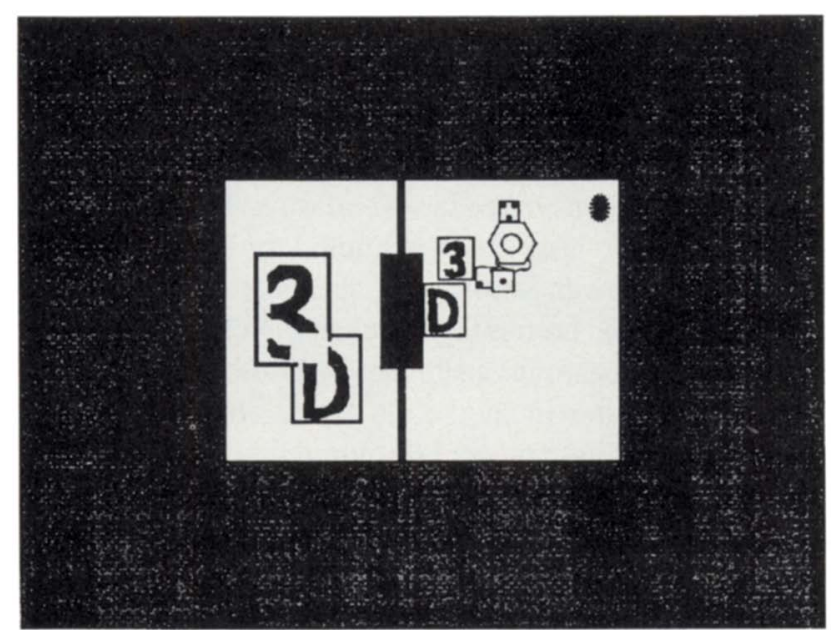

Fig. 3. One perspective of the hologram images, showing three of the four image groups.

visible from the viewpoint selected for Fig. 3; it is hidden behind the right side of the mask. The two dice/hex-nut image groups are located symmetrically with respect to the point illuminating source; the source is represented by the "starburst" at the upper right in Fig. 3. One dice/hex-nut image group $\left(I_{2}\right)$ is just to the left of the illuminating source, as shown; the other $\left(I_{2}^{\prime}\right)$ is immediately to the right of the illuminating point source, but, as noted, is obscured by the mask for the particular view selected.

\section{Activities Using the Classroom Hologram}

Students are first instructed to view the hologram images and to locate all four of the image groups shown in Fig. 2. Students must stand back at least $30 \mathrm{~cm}$ from the hologram or they will miss the real image of the symbols " 3 " and " $D$ " (these stand out clearly and dramatically about $10 \mathrm{~cm}$ in front of the hologram, as shown in Fig. 3).

Students report their preliminary discoveries about the hologram images by making a sketch like Fig. 2 , showing the experimental arrangement and the approximate location of each of the four image groups. In addition to making the drawing, students create a table like Table I to report their findings. In the table students report respectively the name of the image group ("3/D" or "dice/hex-nut"), general location of each image group (same side or opposite side of hologram relative to viewer), and the orientation of each of the four image groups (upright or inverted), using the first three columns of their table. As noted in Table I, students determine that there are two images of the " 3 " and " $D$ " symbols, both relatively close to the hologram, with one image on the same side of the hologram as the viewer and one on the opposite side. In contrast, both of the "dice/hex-nut" images are on the opposite side from the viewer and farther from the hologram.

The four image groups are next classified as real or virtual, depending on whether they can be projected onto a screen; screens are provided in the form of translucent plastic or sheets of typing paper. The screen is placed at the location of the real " $3 / \mathrm{D}$ " image group $\left(I_{1}\right)$; students in this way demonstrate for themselves the process of projecting an image onto a screen. The screen is then used as needed to find other image groups that can be projected onto the screen; this of course provides the basis for classifying each of the four image groups as real or virtual. The definitions of real and virtual images that I prefer are provided to the students in the written instructions. The provided definition of a real image is: an image that can be projected onto a screen. The provided definition of a virtual image is: an image that cannot be projected onto a screen. It is noted that no extra lenses are permitted in applying this test. Many students will, of course, place the screen between the light source and the transmission hologram in their efforts to locate other real images, and rather quickly realize that this is fruitless; this process can produce significant learning. In the fourth column of the table (see Table I) students report their conclusions as to whether each image group is real or virtual.

Students measure the distances from the images to the hologram. The distance to the real image $\left(I_{1}^{\prime}\right.$ in Fig. 2$)$ is measured first; this measurement is made twice using each of two different methods. In the first method the real " $3 / \mathrm{D}$ " image is projected onto a screen and the distance from the hologram to the screen is measured. In the second method parallax is used to place a marker (a stick inserted into a one-hole rubber stopper) at the location of the real image; students then measure the distance from the marker to the hologram. Having been introduced to two methods for determining image locations, students are instructed to measure the distance to the other " $3 / \mathrm{D}$ " image group. Of course only the parallax/marker method can be used for determining the location of these virtual images, but students are simply told to devise a method. They seem able to recognize quickly that no screen can be used and instead select parallax between the hologram and marker as the appropriate method. Students report these distances in the fifth column of the table, which completes Table I.

It is worth noting that measurements of hologram virtual image distances are more easily done than lens virtual image

Table I. Typical information provided by students to summarize hologram image observations.

\begin{tabular}{|c|c|c|c|c|}
\hline $\begin{array}{l}\text { Name of } \\
\text { image group }\end{array}$ & $\begin{array}{c}\text { Location } \\
\text { (relative to viewer) }\end{array}$ & Orientation & Character & $\begin{array}{c}\text { Distance (cm) } \\
\text { (hologram to image) }\end{array}$ \\
\hline " $3 / \mathrm{D} "$ in front & same side & upright & real & $15.5 \pm 0.2$ \\
\hline "3/D" behind & opposite side & upright & virtual & $11.6 \pm 0.2$ \\
\hline dice/hex-nut on left & opposite side & upright & virtual & not required \\
\hline dice/hex-nut on right & opposite side & inverted & virtual & not required \\
\hline
\end{tabular}


distances because both the marker and the hologram image can be viewed directly through the hologram; in contrast, for a lens the image to be measured must be viewed through the lens and the marker must simultaneously be viewed around the edge of the lens; the image of the marker formed by the lens, which is present throughout the measurement, must be consciously ignored by the student, frequently causing confusion.

The final activity required is the creation of ray diagrams explaining the formation of real and virtual images. To introduce the basic form of the required ray diagrams, students are instructed to explore and describe with a ray diagram the light beam coming from the point source to the hologram. Students use a moveable screen to explore the light beam coming from the point source to the hologram; they of course discover that the light beam expands as it travels from the lens to the hologram. Students are also asked to pay some attention to how the lens works and to show this in their diagram. They are then told to create a ray diagram using at least five representative rays to explain the effect of the lens on the laser beam and to show the light beam spreading out as it approaches the hologram; the type of diagram expected is shown in Fig. 4a. Since this is the first ray diagram required in a series of ray diagrams, it is important that students completely understand the type of diagram required before proceeding; discussions with the class to emphasize the presence of both converging and diverging ray bundles in Fig. 4a seem helpful at this point.

Having described the light beam illuminating the hologram, students are asked to create a ray bundle diagram explaining formation of the real image $\left(I_{1}^{\prime}\right)$; they extend the diagram of Fig. 4a to include representative hight rays moving from the hologram toward the real image of the " $3 / \mathrm{D}$ " symbol. Students are instructed to proceed in three steps. First, a point is added to Fig. 4a representing the real image location; this location is represented simply by one example image point, as shown in Fig. 4b. Second, a symbol is added representing the viewer's eye, also shown in Fig. 4b. Finally, five or more light rays are drawn leaving the hologram and passing through the one real image point. Students rather uniformly have good success with this activity, and end up with a diagram much like that shown in Fig. 4b; the main point is that the real image is formed by a converging ray bundle. Having completed this activity it becomes obvious to many students that real images will always be formed on the same side of an imaging system as the viewer. Students are asked to draw a conclusion in this regard. Students do not all articulate their reasoning equally well, and it is useful to encourage discussion of this point.

The process of ray diagram construction is then repeated for a virtual image; specifically students are instructed to consider the virtual image of the " $3 / \mathrm{D}$ " symbol, labeled $I_{1}$ in Fig. 2. The preliminary diagram showing light traveling from the lens to the hologram, shown in Fig. 4a, is drawn again; then light rays leaving the hologram to form the virtual image are drawn. A correct response is shown in Fig. 4c.

Fig. $4 \mathrm{a}$

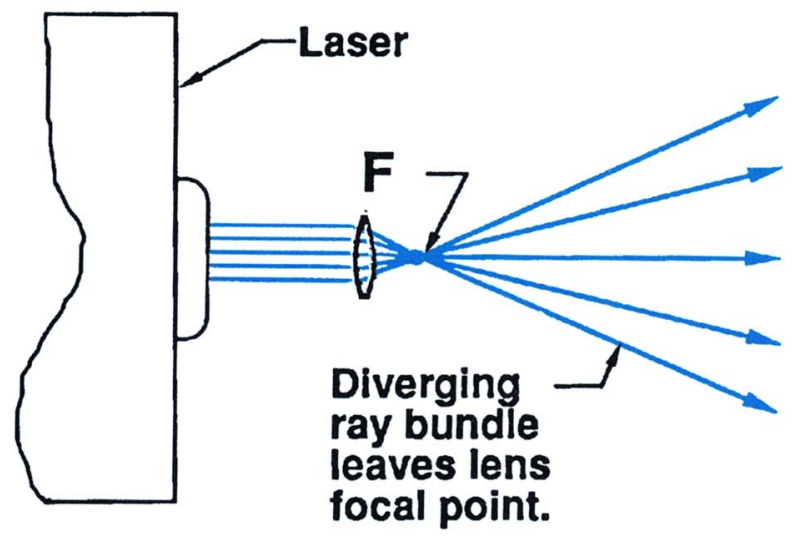

Fig. 4b
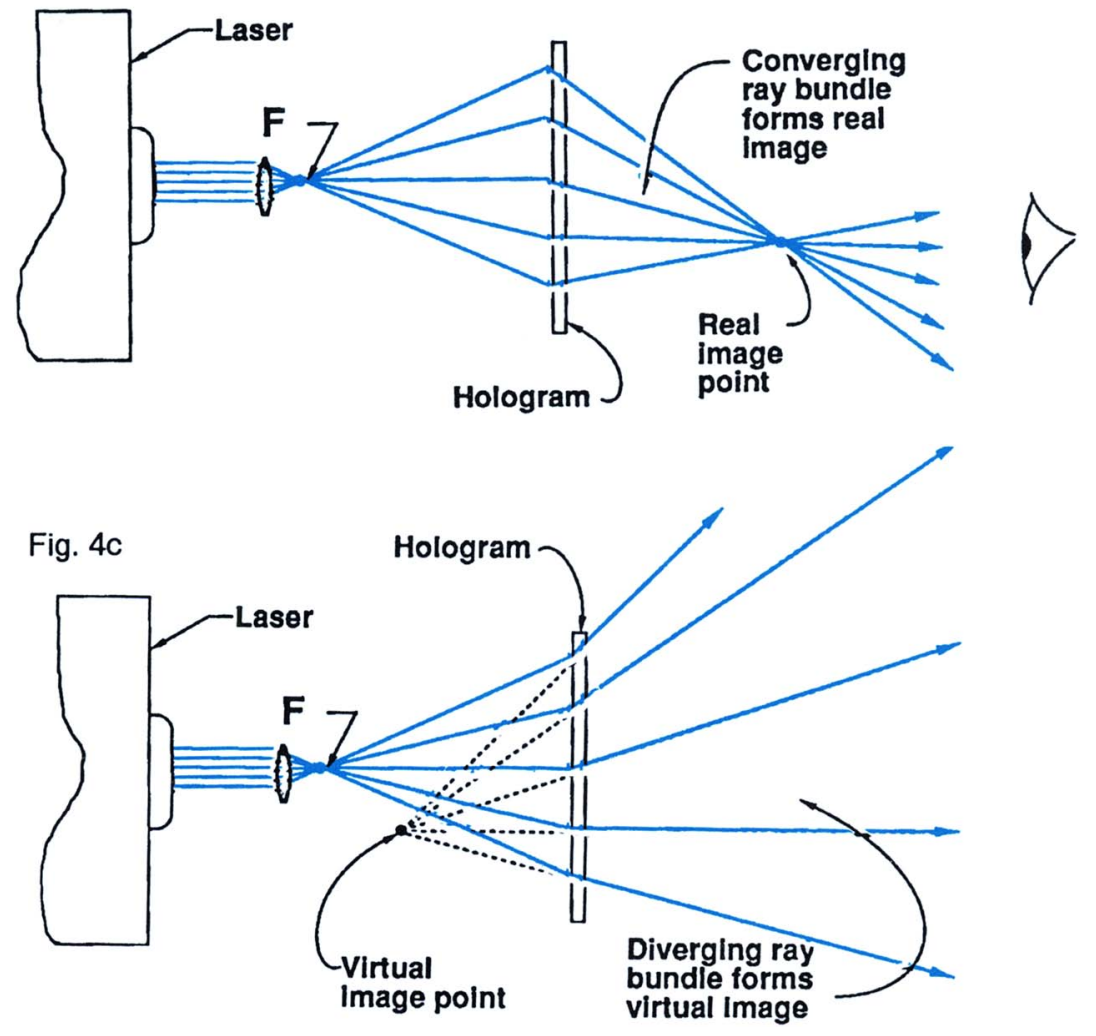

Fig. 4. Figure $4 a$ represents the laser beam illuminating the hologram. Figure $4 \mathrm{~b}$ shows real image formation; a converging ray bundle leaves the hologram. Note the location of the viewer's eye. Figure $4 c$ shows virtual image formation; a diverging ray bundle leaves the hologram. 


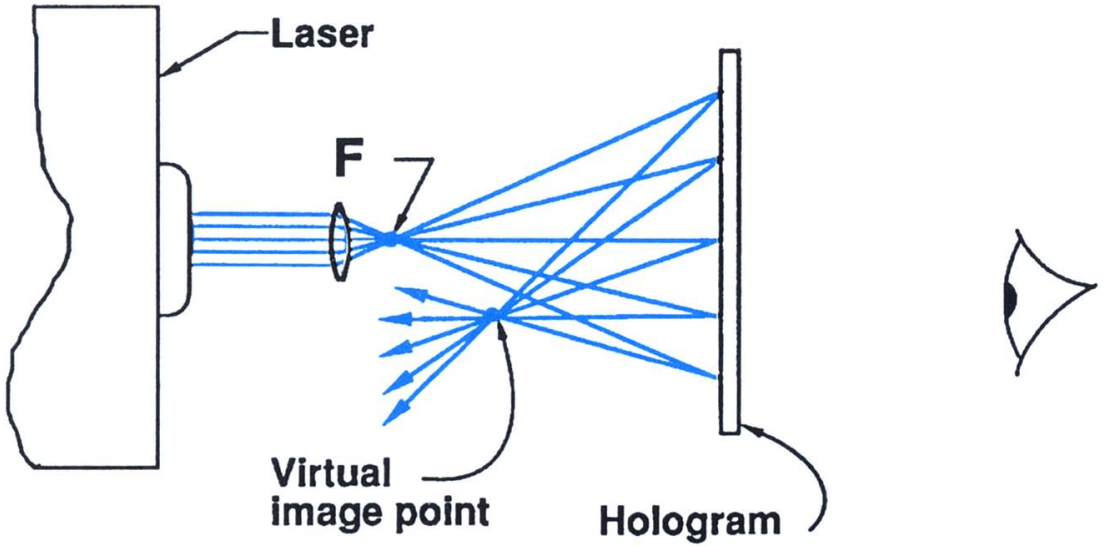

Fig. 5. Type of erroneous diagram drawn by a significant number of students to represent virtual Image formation. Students making this error also tend to omit the symbol for the viewer's eye.

The required ray-drawing activity makes explicit a misconception about virtual images held by many; students frequently draw an erroneous ray bundle diagram like that shown in Fig. 5. Students draw light rays reflecting backwards from the hologram and traveling away from the viewer and toward the virtual image location. This type of drawing and associated student remarks indicate their plausible but erroneous belief that if an image is seen behind the hologram, then light rays must be converging toward the location of that image.

Students and instructors, working together, may be able to exploit this explicit representation of the student's misconception, if ray diagrams like Fig. 5 made by students are immediately discussed with an instructor. Instructors can help students confront inconsistencies in their model; in particular it should be pointed out to the student that light must enter the eye of the viewer, but cannot if it travels backwards toward the virtual image location. Students making a drawing like Fig. 5 also tend to leave the symbol for the viewer's eye out of the drawing. A second clue that can be offered to students is the fact that no image light appears on a screen placed at the location of the image; however this is less persuasive to me, because there is, in fact, a weak real image produced by light reflected backwards from the transmission hologram. This reflected light image cannot, in practice, be seen on a screen because there is too much background light due to the raw laser beam. The fact that such a real image due to reflected light can (and does) exist emphasizes that the students" "erroneous" diagram is in fact very plausible, and that students should not be scorned for making a drawing like Fig. 5. Although my purpose in developing these activities was not to do formal "misconceptions" research, it is interesting to compare the outcomes of these activities with earlier work by Goldberg and McDermott relating to student understanding of images formed by mirrors and lenses. ${ }^{4}$

As another brief activity, our students view the image of a skull formed by a reflection hologram taken from a magazine advertisement created by the Polaroid corporation. ${ }^{5}$ This skull image is, in my judgement, remarkable in its bright clear appearance, making it of interest in its own right; however, the skull image also has special value for this lab because the image lies partly in front of the film surface, and partly behind, making parts of the image real and other parts virtual. Students see a single continuous image that has both real portions and virtual portions within the same image; recognizing that this is possible provides a useful review and generalization of the relationship between image character (real or virtual) and image location (on the same side as viewer or on the opposite side from viewer).

Students doing these activities work from a four-page lab instruction. A copy of this lab instruction is available on request.

\section{How to Make a Classroom Hologram}

This description of how to make the classroom hologram is intentionally brief, but should be useful to persons already

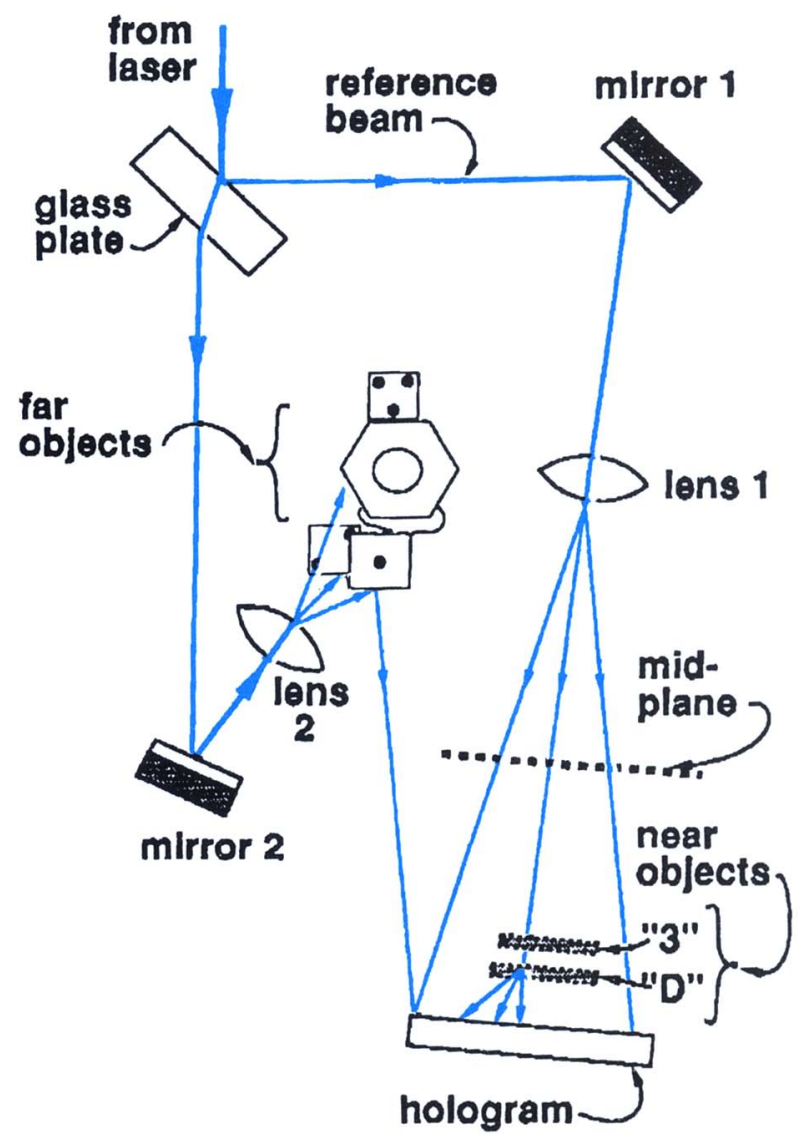

Fig. 6. Key details of the experimental arrangement used to form the classroom hologram. Also shown is the "midplane," useful in predicting the character of the Images that will be formed by the hologram. 
having a basic familiarity with holography. The experimental arrangement is shown in Fig. 6.

To make a classroom hologram we create two object groups, one close to the hologram and one farther away. We select both "near" and "far" objects so that both real and virtual images will be formed by the resultant hologram. Whether the images formed by the hologram will be real or virtual is controlled in part by the relatively little known "midplane rule." The midplane nule refers to a plane, shown in Fig. 6, parallel to the plane of the hologram and halfway between the reference beam lens and the hologram; the rule says that objects closer to the hologram than the midplane will form one virtual and one real image, whereas objects more distant than the midplane will form two virtual images. In our case the far object group is a collection of dice and a hex-nut. The near object group is a " 3 " and a " $D$ " symbol. The midplane rule assumes that the hologram is viewed with a beam identical to the reference beam used to form the hologram.

The two object groups scatter light to the hologram; this light comes from a helium-neon laser having light power between one and five milliwatts. The laser beam is divided into two beams by a thick glass plate. One beam passes straight through the glass plate, spreads after leaving lens 2 and illuminates the far object group. The other beam, labeled "reference beam" in Fig. 6, reflects off the surface of the glass plate, is spread by lens 1 and back-lights the near object group. However, the beam passing through lens 1 serves a double purpose: in addition to back-lighting the near objects, most of this beam goes directly to the hologram without scattering from an object; hence the name reference beam.

The near objects, a " 3 " and a " $D$ " symbol shown in Fig. 3 , are dry transfer figures (available in art supply stores) affixed to translucent glass plates; because the glass plates are back-lit, the transfer figures are seen in silhouette. The near glass-plate objects cast a shadow onto the hologram creating a "window," a clear region near the center of the hologram; this "window" occurs because no two-beam light wave interference pattern is present in the shadowed region during hologram recording. As mentioned previously this "window" is blocked with an opaque black rectangle, as shown in Fig. 3, to prevent glare. The near objects form one virtual image (coincident in location with the original " 3 " and " $D$ " objects) and one real image, in agreement with the midplane rule.

For the "far object," any light-colored opaque object can be used; we chose dice and a white spray-painted hex-nut. Also in agreement with the midplane rule, the far object group forms one virtual image located coincident with the original objects and a second virtual image group that turns out to be on the other side of the lens 1 from the original object group. Only one of these two virtual image groups is visible in Fig. 3, but both are shown in Fig. 2.

\section{Summary and Discussion}

The hologram-based teaching activities described here are guided explorations that emphasize the following topics:

\section{SUPERCONDUGTING BATTERY KIT}

\author{
(NEW)
}

- EXPLORE THE PHYSICS OF THE BATTERY OF THE FUTURE

- STORE ELECTRIC CURRENT IN A SUPERCONDUCTING TOROID

- CALCULATE THE LENGTH Of BATtERY LIFE (1023 YEARS)

- DESIGNED TO MIMIC THE U.S. NAVY'S ADVANCED SMES(SUPERCONDUCTING MAGNETIC ENERGY STORAGE) DEVICE PLANNED FOR POWERING SPACE-BASED LASERS

CATALOG TEL $380 \quad \$ 175.00$

\section{TEL-Atomic ${ }^{\text {sm }}$ \\ Incorporated}

P.O. Box 924 O Jackson, wl 49204

(517) 783-3039 ○ 1-800-622-2866 O Fax (517) 783-3213

projecting images onto a screen and using this as a test for distinguishing real from virtual images; measuring image locations by parallax; and explaining real and virtual images using converging and diverging ray bundles diagrams. The activities can identify a common student misunderstanding about virtual image formation; immediate interaction with a teaching assistant is important in overcoming the misunderstanding, once it is made explicit.

(It should be noted that the definition of real/virtual images used in these activities is not the most fundamental definition and should be used with some caution. Whether or not an image can be projected onto a screen should not be promoted as the fundamental distinction between real and virtual images. In particular, pinhole images can also be projected onto a screen, but are neither "real" nor "virtual." A correct experimental procedure for identifying real images is to note that when viewed directly without the aid of a screen they must be on the same side of the imaging system as the viewer and also that they must have a definite location. Virtual images are on the opposite side from the viewer and also have a definite location. Images that can be projected onto a screen, but which have no definite location are pinhole images, i.e., images formed by limiting the diameter of the 
exit pupil of the imaging system. However, for the purposes of the elementary activity described in this article, simply emphasizing that real images can be projected onto a screen whereas virtual images cannot seems adequate.)

These hologram image explorations are new in two ways. First, numerical predictions of image distances, although taught in a separate lab activity, are not taught here; instead, a qualitative treatment of converging and diverging rays bundles is emphasized. Second, these activities rely on the use of hologram images. Although I had originally thought of the image-forming system, a hologram, as being the most important new characteristic in these activities, I am now coming to believe that the particular qualitative guided explorations that we have discovered are a more significant new aspect. I have seen no such activities reported for lenses or mirrors, although it would seem to be possible, in principle, to create such activities.

Our method of teaching about images can be compared with three other possible approaches. First would be the traditional quantitative treatment of lens (and/or mirror) images; ${ }^{1}$ the second, quantitative treatments of hologram images, ${ }^{2,3}$ closely resembles the methods used for lenses; the third would be a qualitative treatment of lens (and/or mirror) images analogous to the hologram activities just described, but which seems not to have been developed or used anywhere. Following is a brief comparison of each of these approaches to our hologram method.

The first method, based on quantitative treatments of lens (and/or mirror) images, is well known and widely appreciated, and its advantages will not be reviewed here; this quantitative approach cannot be replaced by the activities described here, but these two methods may complement each other. A negative aspect of the traditional quantitative lensbased methods is that they can simply become a memorized, but poorly understood, procedure for obtaining an answer to a particular word problem. It is my belief that the typical brief exposure of beginning students to such quantitative methods alone will not significantly improve the performance of students in qualitatively representing a virtual image with a correct diagram (Fig. 4c) rather than an incorrect diagram (Fig. 5). A second negative aspect of the usual approach of emphasizing mainly lens images is that particular characteristics of lens images (e.g., real lens images are inverted) may be assumed to be identifying characteristics of all real images. The more qualitative guided explorations described in this article provide a complementary teaching tool that hopefully can deepen student understanding; even the best students will enjoy the activities presented here and benefit from them. For weaker students, seeing the hologram images might stimulate additional interest.

The second approach mentioned, based on quantitative predictions of hologram image location, is not as widely known or used as quantitative lens methods. Quantitative thin-hologram models teach about interference and diffrac- tion rather than refraction; the image location equations are based on the thin-grating equation rather than Snell's law. The main obstacle to introducing these models into introductory physics courses seems to be lack of time and the fact that these methods are not described in introductory texts. Quantitative hologram models can be expected to have advantages and disadvantages similar to those associated with the elementary quantitative lens methods.

The third method, using lens (and/or mirror) images in a manner analogous to the use of hologram images described here seems not to have been developed. One reason for this is that, in practice, it is more difficult to simultaneously form real and virtual images with lenses than with holograms. Also, as already explained, measuring virtual image locations with lenses is more difficult than with transmission holograms.

My preference is to use both the quantitative and qualitative approaches, to use both lens and hologram images, and to a lesser extent, to utilize curved mirror images. We have traditionally taught quantitative models of imaging using lenses and mirrors in our introductory physics course, and we will continue this approach; quantitative models of hologram imaging will probably be reserved for a sophomore-level optics lab course. However, the qualitative hologram image activities we've described require only about one hour of lab time. I encourage physics teachers to try using holograms to teach about real and virtual images.

\section{Acknowledgment}

I have received many suggestions from physics teachers who have participated in PRISMS workshops at the University of Northern Iowa. These workshops were organized by Dr. Roy Unruh and Dr. Tim Cooney. Several teachers have tried these activities in their own schools and have given me feedback. I especially appreciate the responses received from Wade Sick of Red Oak High School, Red Oak, IA, Jan Mader, Great Falls High School, Great Falls, MT, and Doug Nauman, Washington High School, Cedar Rapids, IA.

\section{References}

1. See for example Hillard K. Macomber, Introductory Physics Laboratory Manual, 3rd. ed. (Burgess International Group, Inc., Bellwether Press Division, Minneapolis), pp. 52-55.

2. N. Abramson, The Making and Evaluation of Holograms (Academic Press, London, 1981). See especially p. 55, Fig. 2.12. See also: Henri H. Arsenault, "Cardinal Points and Principal Rays for Holography," which is Chapter 7 in Handbook of Optical Holography, edited by H.J. Caulfield (Academic Press, New York, 1979), pp. 239-256.

3. D.W. Olson, "The Abramson ray-tracing method for holograms," Am. J. Phys. 57, 5 (1989), pp. 439-444; also "The elementary plane-wave model for hologram ray tracing," pp. 445-455.

4. F.M. Goldberg and L.C. McDermott, Phys. Teach. 24, 472 (1986); Am. J. Phys. 55, 108 (1987).

5. This skull reflection hologram has recently become available from Arbor Scientific, P.O. Box 2750, Ann Arbor, MI 481062750; 1992 Catalog Item \#P2-7051. 